\title{
A Novel Optical Carrier Phase Estimation Technique Based on Viterbi-Viterbi and QPSK Partitioning for 16-QAM
}

\author{
Heba M. Shehata, and Ziad A. El-Sahn \\ Photonics Group, Electrical Engineering Department, Alexandria University, Alexandria 21544, Egypt \\ Email: h.m.shehata@ieee.org. ziad.elsahn@ieee.org
}

\begin{abstract}
We propose a novel carrier phase estimator based on two-stage Viterbi-Viterbi and QPSK partitioning for 16-QAM. Simulation shows that the proposed algorithm is highly tolerant to laser linewidth with a reduced cycle slip probability.

OCIS codes: (060.2330) Fiber Optics Communications; (060.1660) Coherent Communications
\end{abstract}

\section{Introduction}

To cope with the exponential growth in the data traffic, together with a minimum transmission cost imperative, spectrally-efficient modulation techniques have recently been combined with coherent optical communications rather than intensity-modulation direct detection [1]. The integration of digital signal processing (DSP) techniques into the optical receiver has also received much attention in order to fully retrieve the transmitted data.

Optical carrier phase fluctuations due to the finite laser linewidth is one of the strongest challenging impairments that face the coherent detection of the received optical signal and mandate the use of carrier phase recovery (CPR) stage at the optical receiver DSP stack [1]. One of the commonly used CPR techniques is Viterbi-Viterbi (VV) algorithm. It is a non-data-aided (NDA) or blind CPR algorithm that was commonly used with M-ary phase shift keying (M-PSK) modulation and recently with M-ary quadrature amplitude modulation (M-QAM) when combined with PSK-partitioning techniques [2]. Although Viterbi-Viterbi algorithm is a simple linewidth-tolerant algorithm, it suffers from cycle slips when the phase changes abruptly causing a rotation of the constellation axes and hence degrade the performance of the carrier phase estimation (CPE) algorithm significantly [3].

In this work, we propose a new two-stage Viterbi-Viterbi scheme based on two different PSK-partitioning techniques for 16-QAM constellation. The main goal is to achieve high tolerance to the optical carrier linewidth which, in turn, facilitates the implementation of the optical transmitter using commercially available optical sources. The proposed phase estimator consists of two Viterbi-Viterbi stages, the first one utilizes VV algorithm with QPSK partitioning as a coarse estimation stage and the second one is based on a modified VV algorithm proposed in [4] for residual phase errors estimation. Simulations show that the proposed CPE algorithm outperforms VV algorithm for QPSK-partitioned 16-QAM and the modified VV from the linewidth point of view with a noticeable robustness against cycle slips especially at low SNR levels with a slightly increased computational complexity.

\section{Proposed CPE-VV Algorithm}

Because of the phase noise non-stationary stochastic nature, the CPE may suffer from some instabilities especially in high linewidth or low SNR such as cycle slips [3]. Cycle slips may have a catastrophic impact on the estimation process due to the rotation of the estimated constellation points by multiples of $\pi / 2$. This paper proposes a new CPE scheme that has a high linewidth tolerance with lower susceptibility to the cyclic slips. The proposed algorithm consists of two cascaded stages as shown in Fig.1.

The first stage is a VV stage using QPSK partitioning that uses part of the incoming window symbols for the phase estimation and then the phase estimate is applied in a window-fashion to all of the data symbols [5]. This stage, in fact, acts like a coarse estimation stage that reduces the probability of cycle slips significantly especially at low SNR values and eliminates any residual frequency offset from the carrier frequency estimation (CFE) previous stage.

The second stage is the modified VV estimator in [4] in which the constellation points are classified, rotated and a power of 8 is used to remove the used modulation from all data symbols for estimation. This stage uses all the data symbols in the estimation process according to class transformation (CT) process and it is used as a residual phase error estimator that increases the linewidth tolerance of the scheme.

Fig. 1 also shows a simple constellation diagram of the used symbols in each estimation stage where the incoming data symbols are partitioned into class $1\left(\mathrm{C}_{1}\right)$ and class $2\left(\mathrm{C}_{2}\right)$ symbols. Only $\mathrm{C}_{1}$ symbols are used for the coarse estimation and then all $C_{1}$ and $C_{2}$ symbols are fed to the modified VV with $C_{1}$ symbols rotated by $\pi / 8$ as in [4] for fine estimation. 


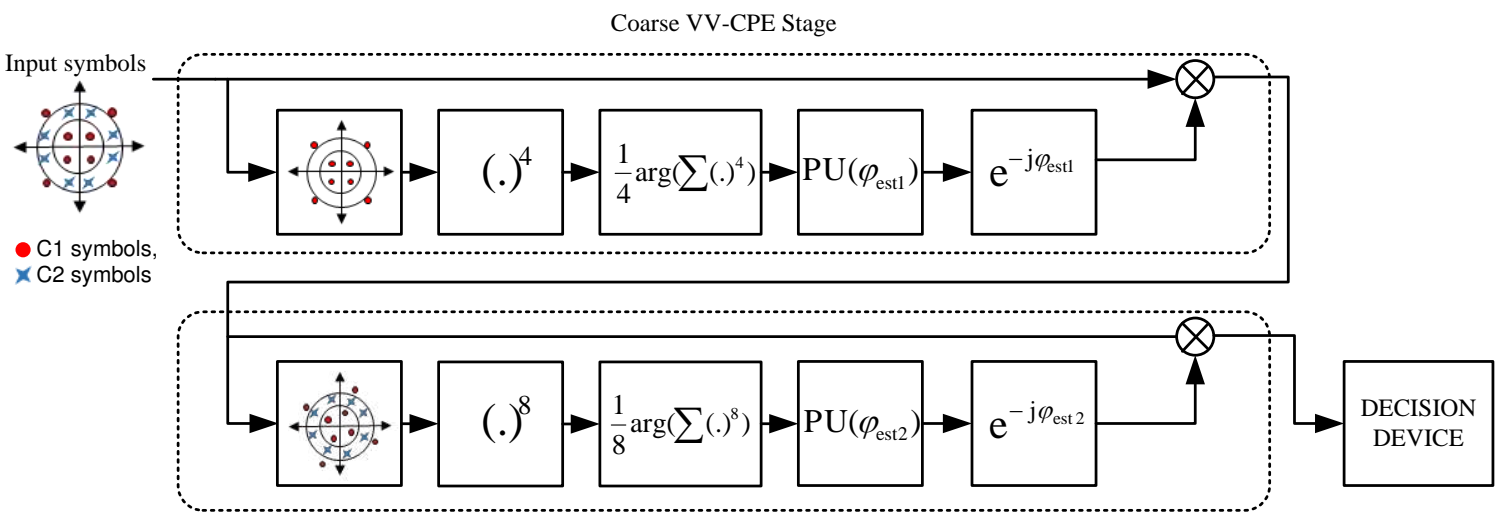

Fine VV-CPE Stage

Fig. 1. The proposed two-stage Viterbi-Viterbi algorithm based on QPSK partitioning and modified VV with class 1 and class 2 symbols highlighted, PU (.) is a phase-unwrapping stage and $\left(\varphi_{\text {est }}\right)$ is the estimated phase error.

\section{Simulation Results and Discussion}

The simulation results are obtained using 16-QAM data symbols and the received signal is assumed to be sampled with perfect timing recovery, and the optical channel impairments are equalized so that only the impact of the phase noise is studied. The phase noise is modeled as Wiener process

$$
\varphi_{\mathrm{n}}=\sum_{\mathrm{i}=-\infty}^{\mathrm{n}} \mathrm{u}_{\mathrm{i}}, \quad \varphi(\mathrm{n}+1)=\varphi(\mathrm{n})+\mathrm{u}_{\mathrm{n}}
$$

Here $u_{i}$ is a Gaussian random variable with zero mean and a variance of $2 \pi \Delta v T_{s}$ where $\Delta v$ is the optical source linewidth and $\mathrm{T}_{\mathrm{s}}$ is the data symbol duration.

Fig. 2 shows the performance of the proposed scheme against the laser linewidth $x$ symbol duration product. The performance of the proposed algorithm is compared to the conventional VV algorithm using QPSK partitioning with a maximum likelihood (ML) estimator as a second stage, and the modified VV-CPE with ML as a second stage at a bit error rate (BER) of $10^{-3}$. The BER of $10^{-3}$ corresponds to the threshold of the subsequent forward error correction (FEC) stage of the receiver.

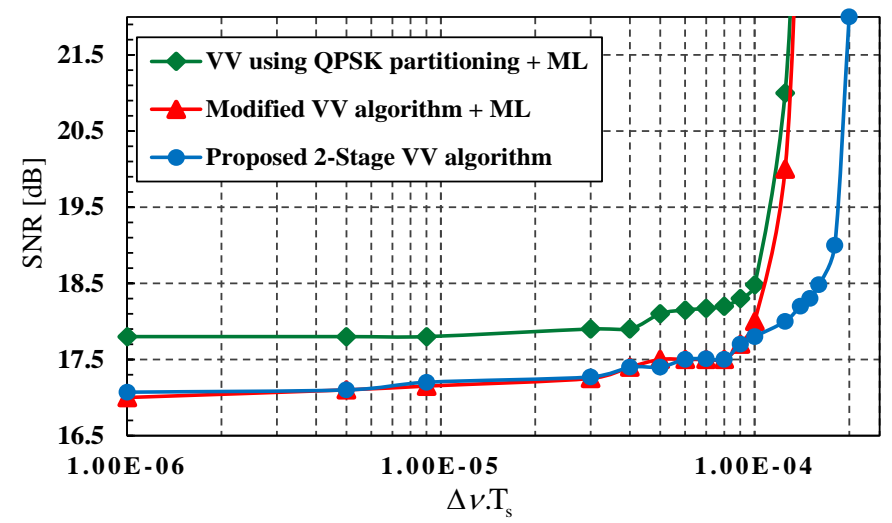

Fig. 2.: Linewidth tolerence of the proposed 2-stage VV CPE compared to VV using QPSK partitioning and modified VV at BER of $10^{-3}$.

The cycle slips probability for the proposed algorithm compared to VV with QPSK partitioning and the modified $\mathrm{VV}$ algorithm is shown in Fig. 3. Results show that the proposed scheme is more linewidth tolerant than the other two systems in addition to a noticeable lower cycle slips probability than the modified VV-CPE.

At BER of $10^{-3}$, it can be shown that the higher achievable values of the linewidth tolerance are of $8.8 \times 10^{-5}$ and $10^{-4}$ for VV-CPE using QPSK partitioning with ML and for the modified VV-CPE with ML, respectively, while the proposed algorithm achieves $1.6 \times 10^{-4}$, all at $1-\mathrm{dB}$ SNR penalty. This linewidth tolerance is achievable with more than $22 \%$ reduced probability of cycle slip occurrence than the modified VV algorithm at SNR of $17.3 \mathrm{~dB}$. The window 
size of each stage was optimized to be $\left(\mathrm{N}_{1}=\mathrm{N}_{2}=20\right)$ which corresponds to the highest tolerable linewidth at less than 1-dB power penalty as illustrated in Fig. 4. From the computational complexity point of view, the proposed algorithm needs 1.56 times more real computations (additions and multiplications) than the modified VV with ML [7] at the selected window size.

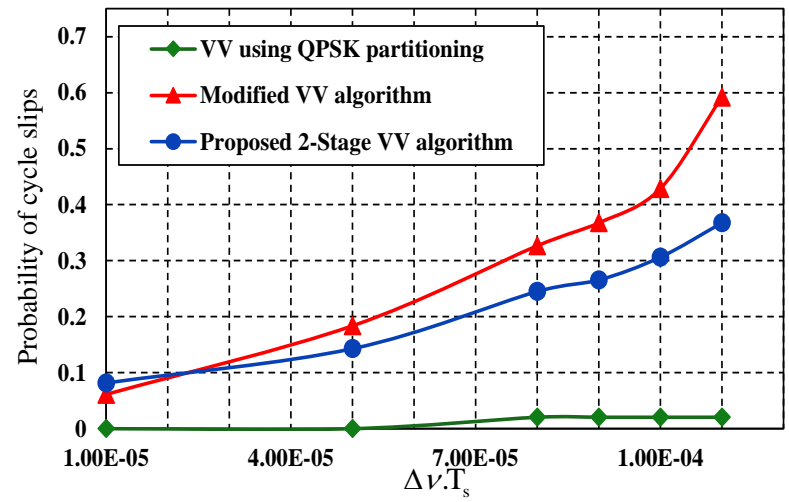

Fig. 3. The probability of occurrence of the cycle slips of the proposed 2-stage VV compared to VV using QPSK partitioning and the modified VV at BER of $10^{-3}$ and SNR of $17.3 \mathrm{~dB}$.

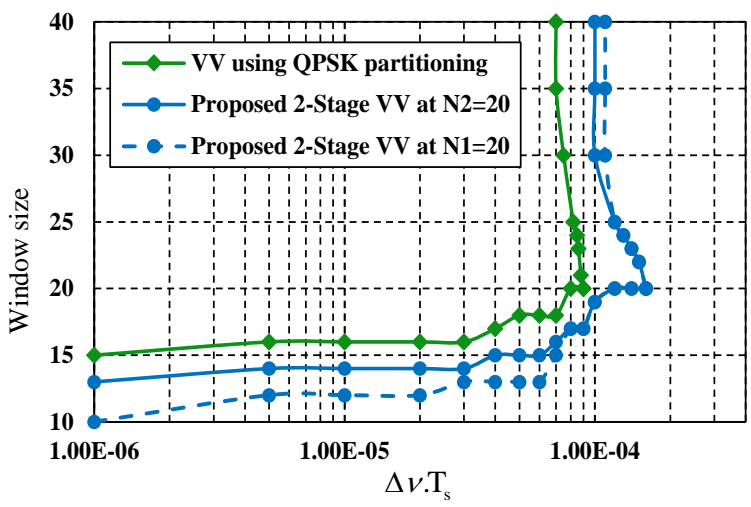

Fig. 4. The averaging window size of VV algorithm versus the linewidth at BER of $10^{-3}$ and less than 1-dB optical power penalty.

\section{Conclusion}

We reported a novel two-stage VV-CPE scheme for 16-QAM. The proposed algorithm shows a high linewidth tolerance with a noticeable cycle slip reduction. The algorithm performance was compared to VV algorithm using QPSK partitioning with ML and the modified VV with ML.

\section{References}

[1] S. Savory, et al., "Digital Coherent Optical Receivers: Algorithms and Subsystems", J. Sel. Topics Quantum Electron., 16, 1164 (2010).

[2] I. Fatadin, et al., "Laser Linewidth Tolerance for 16-QAM Coherent Optical Systems Using QPSK Partitioning", Photonic Technology Letters, 22,631 (2010).

[3] A. Bisplinghoff, et al, "Slip-Reduced Carrier Phase Estimation for Coherent Transmission in the Presence of Non-Linear Phase Noise", Proc. OFC'13, OTu3I.1, (2013).

[4] S. Bilal, et al., "Dual Stage Carrier Phase Estimation for 16-QAM Systems Based on a Modified QPSK-Partitioning Algorithm ", Proc. ICTON'15, We.D1.2 (2013).

[5] Jian Hong et al., "A simple three-stage carrier phase estimation algorithm for 16-QAM systems based on QPSK partitioning and maximum likelihood detection ", Proc. OFC'13, OTu3I.5 (2013).

[6] Y. Chin et al., "Low-Complexity Joint Frequency Offset and Carrier Phase Estimation Based on QPSK Partitioning for DP 16-QAM", Optics Communications, 342, 44 (2015).

[7] S. Bilal, et al., "Performance and Complexity Comparison of Carrier Phase Estimation Algorithms for DP-64-QAM Optical Signals", Proc. ECOC'2014, P.3.11 (2014). 